\title{
Assessment of early retinal changes in diabetes using a new multifocal ERG protocol
}

\author{
Yoshiaki Shimada, Yong Li, Marcus A Bearse Jr, Erich E Sutter, Wayne Fung
}

\begin{abstract}
Aims-To assess early functional retinal changes in diabetics without retinopathy, a new multifocal stimulus paradigm was used that emphasises fast adaptive response contributions.

Methods-25 normal control subjects (25 eyes) and 11 diabetics without retinopathy (22 eyes) served as subjects. Stimulation and analysis were performed with Veris Science 4.0. A stimulation protocol was used that combines regular multifocal flicker stimulation with a periodic "global" flash inserted between the multifocal stimuli. The multifocal stimuli were presented four video frames apart. The global flash covered the entire screen in the third frame of the four frame interval. The remaining two frames were dark. The periodic global flashes could only contribute to the focal responses if they were affected by the multifocal stimulation. A non-linear component induced by the interaction of the focal and global flashes was observed. The differences between control subjects and diabetics were assessed in both the multifocal responses and their induced effect on the following global flashes.
\end{abstract}

Results-The responses to focal flashes were reduced significantly in diabetics matched in age to the control subjects. The induced components showed large intersubject variability in controls and patients, and did not differ significantly between the two groups.

Conclusion-The periodic global flashes produce a greater multifocal response reduction in diabetics than in normals, indicating impairment in the rate or magnitude of recovery from the bright preceding stimulus. The new stimulation protocol reveals early changes in retinal function of diabetics.

(Br F Ophthalmol 2001;85:414-419)

The California Pacific Medical Center, San Francisco, California, USA

W Fung

Correspondence to: Yoshiaki Shimada, Department of Ophthalmology, Fujita Health University, 1-98 Dengakugakubo,

Kutukake-cho, Toyoake-city, Aichi 470-1192, Japan

ysmd@za2.so-net.ne.jp

Accepted for publication 6 October 2000

While the conventional electroretinogram (ERG) can reveal early functional changes in the retinas of diabetics, it has been controversial as a clinical predictor of retinopathy. Many reports ${ }^{1-6}$ investigating oscillatory potentials (OPs) and/or pattern responses suggest that ERG abnormalities occur before any signs of retinopathy. However, while such abnormalities were evident in more advanced stages of diabetic retinopathy, the ability of the ERG to detect changes in subclinical cases has been questioned. $^{78}$
During the past decade, the multifocal ERG ${ }^{9}$ appeared as a new technique for exploring human retinal function including diabetic retinopathy..$^{11}$ Through the topographic distribution of first and higher order response components it provides detailed information on the local non-linear response properties of the retina. The usefulness of this information for the diagnosis of retinal disease such as diabetic retinopathy needs to be fully explored.

Recordings made by Palmowski et $a l^{10}$ using the standard (fast) multifocal flicker protocol, suggested that second order response components are more sensitive for the detection of early changes in retinal function of diabetics than the commonly considered first order kernel. When derived by means of the standard protocol, the first slice of the second order kernel represents the degree to which the retinal response is affected by an immediately preceding stimulus. This response component can thus reflect the effects of fast adaptive mechanisms. The finding of Palmowski et $a l^{10}$ suggests that recovery of sensitivity following a flash is abnormal in diabetes, even before retinopathy. We focused on the interactions of flashes, using a recently proposed new stimulus paradigm ${ }^{12}{ }_{13}$ that emphasises fast adaptive effects.

\section{Methods}

Twenty five healthy volunteers (control subjects, 19-85 years old, mean 44.1 (SD19.9)) and 11 diabetics (24-69 years old, 49.8 (13.3)) served as subjects. The diabetics had histories of 1-20 year duration of IDDM (insulin dependent diabetes mellitus; six cases) or NIDDM (non-insulin dependent diabetes mellitus; five cases). The non-dominant eye of control subjects (25 eyes) and both eyes of patients (22 eyes) were tested. All subjects underwent ophthalmoscopic examinations and any fundus abnormalities resulted in exclusion from this study. Their corrected visual acuities were $20 / 25$ or better. Pupils were dilated maximally with eye drops of $1 \%$ tropicamide and $2.5 \%$ phenylephrine hydrochloride. The tenets of the Declaration of Helsinki were followed
STIMULATION

Multifocal stimulation and analysis were performed with Veris Science 4.0 (ElectroDiagnostic Imaging, San Mateo, CA, USA). The stimulus was displayed on a high luminance monochrome CRT with P104 (white) phosphor (Nortech Imaging Technologies, Plymouth, MN, USA). A stimulus array of 103 densely packed hexagons (Fig 1A) covered the central $50^{\circ}$. To achieve approximately equal 
A

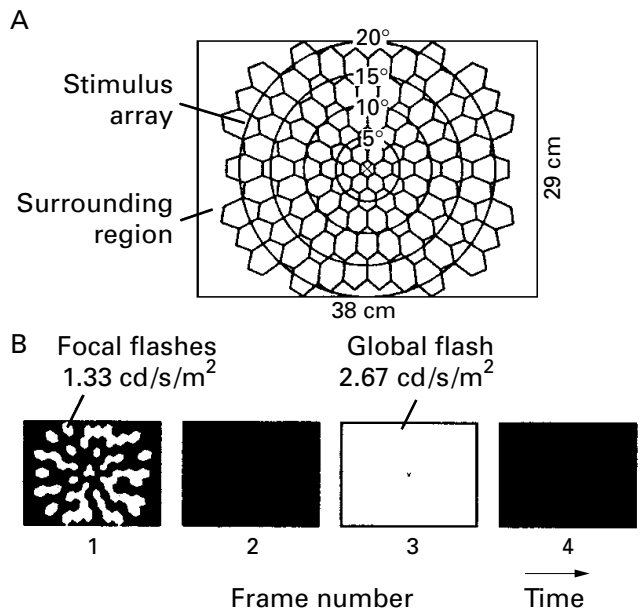

Figure 1 (A) The stimulus, displayed on a $38 \times 29 \mathrm{~cm}$ CRT screen, consisted of 103 hexagons (stimulus array) and a surrounding region. A small fixation cross was located in the centre. (B) Each m-sequence step consisted of four video frames. In frame 1, there was either a flash (1.33 $\left.\mathrm{cd} / \mathrm{s} / \mathrm{m}^{2}\right)$ or darkness, determined by a binary $m$-sequence. The display was dark in frame 2 . In frame 3 , all hexagons and the surrounding region were uniformly flashed $(2.67$ $\left.\mathrm{cd} / \mathrm{s} / \mathrm{m}^{2}\right)$. The display was dark again in frame 4.

A

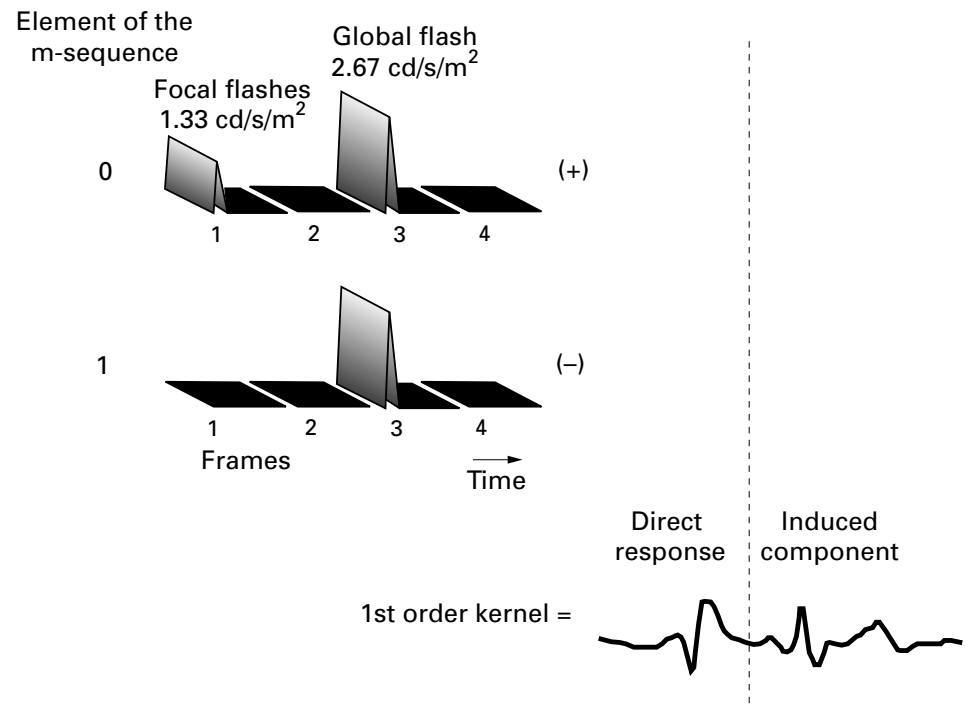

B

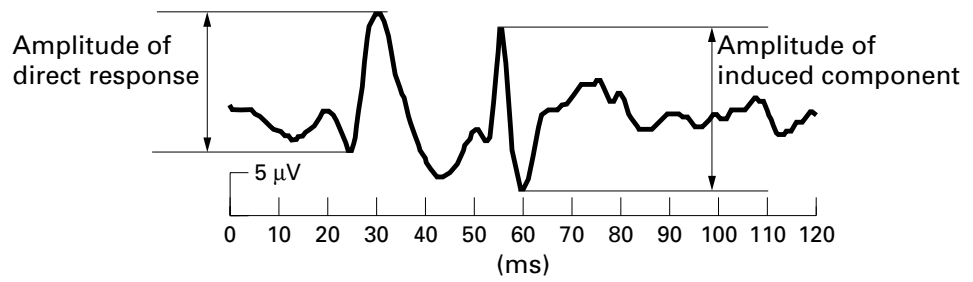

Figure 2 (A) Schematic illustration of the derivation of the first order kernel. It is derived as the cross correlation between stimulus sequence and the response. This computation is equivalent to averaging all the response epochs following a focal flash (top row) and subtracting the average of all those following a stimulus interval without a flash (second row). The resulting average below contains two components: the direct response generated by the focal flash and the effect of the focal flash on the following global flash response $26.7 \mathrm{~ms}$ later (induced component). Measurement of the peak to peak amplitudes of the two components is illustrated in (B). signal to noise ratios at all locations, the size of the hexagons was appropriately scaled with eccentricity $^{9}$ (the stimulus picture stretch factor was 10.46).

The changes of local luminance within each step of the m-sequence stimulus are illustrated in Figure 1B. The duration of each stimulation step was four video frames or $53.3 \mathrm{~ms}$ (at frame rate of $75 /$ second). The first frame in each step consisted of an m-sequence modulated multifocal flash whereby each stimulus patch was either flashed at an intensity of $1.33 \mathrm{~cd} / \mathrm{s} / \mathrm{m}^{2}$ or it remained dark (below $0.01 \mathrm{~cd} / \mathrm{s} / \mathrm{m}^{2}$ ). During the second frame the display screen remained dark (below $0.01 \mathrm{~cd} / \mathrm{s} / \mathrm{m}^{2}$ ). During the third frame the entire screen was flashed at an intensity of $2.67 \mathrm{~cd} / \mathrm{s} / \mathrm{m}^{2}$. This is referred to as the "global" flash. The display was dark again in the fourth frame. Focal and global flash intensities were selected on the basis of data we report elsewhere.

RESPONSE RECORDING

Retinal responses were derived from the cornea by means of a Burian-Allen bipolar contact lens electrode, amplified (50 000 gain) and band pass filtered $10-300 \mathrm{~Hz}$ as in previous studies. ${ }^{10}$ A notch filter was not used, since no power line interference was observed, For patient comfort the records were acquired in 16 segments, each 27 seconds in length. A refractor/camera (Electro-Diagnostic Imaging, San Mateo, CA, USA) was used to refract the subjects and to monitor eye position, alignment of the contact lens electrode on the pupil, and fixation stability during recording. If fixation was lost or a blink or misalignment occurred, the segment was interrupted, discarded, and re-recorded.

\section{RESPONSE ANALYSIS}

Kernels were extracted in the usual way by means of the fast m-transform algorithm. A single cycle (one iteration) of artefact removal ${ }^{9}$ was applied to each record to minimise the effects of occasional blinks or small eye movements. To improve the signal to noise ratio, a small amount of spatial filtering was applied: each trace was averaged with $17 \%$ of the response of its six neighbours.

This study concerns the first order kernel obtained with a global flash paradigm. This kernel represents all response features that directly correlate with the focal stimulation, including second order effects induced on the following global flash response (Fig 2A). Since the responses to the periodically occurring global flashes are added and subtracted the same number of times in the derivation of the kernel, they would cancel completely if the focal flashes did not modify them. However, a considerable response contribution is observed at the proper latency after the global flash (see example in Fig 2B). Thus, this contribution to the first order kernel is a pure non-linearity representing the effect each focal flash has on a following global flash. ${ }^{13}$ To distinguish it from the "direct response" evoked by the focal flashes at about $0-50 \mathrm{~ms}$, this feature of the first order kernel will be referred to as the 


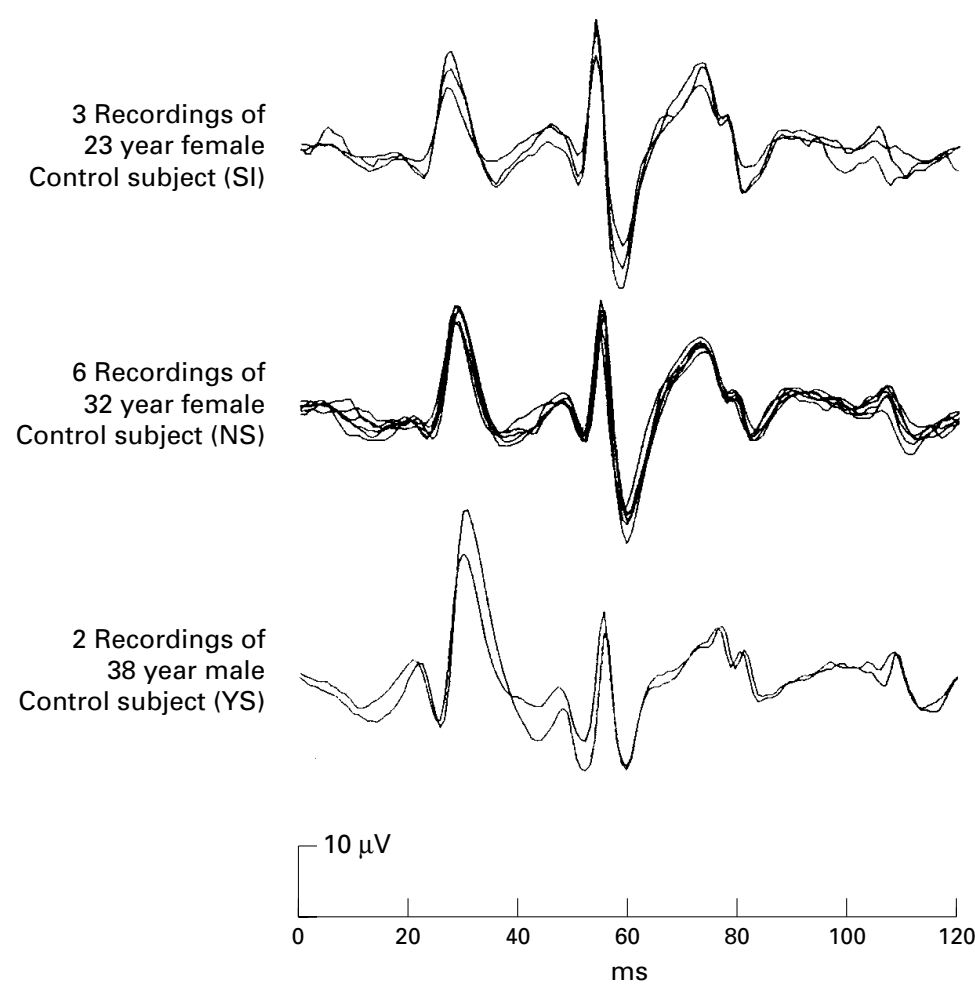

Figure 3 Three subjects were recorded several times on different days with the same eye and contact electrode. The traces shown in the figure represent the first order responses, summed over all 103 hexagons. The six traces shown in the centre panel were derived from a 32 year old female in six recordings spread over the period of 1 month.

"induced component". We measured the amplitudes of the largest peaks of the direct response and the induced component as shown in Figure 2B, and compared these between patients and controls using the two tailed Student's $t$ test.

\section{Results}

In order to test the reproducibility of the results we performed repeated recordings on three of the control subjects (Fig 3). In all cases, the same eye and the same electrode were used on different days. The examples demonstrate excellent reproducibility.

EXAMPLES OF DATA SETS FROM A CONTROL

SUBJECT AND A PATIENT

Sample data sets from a control subject and a patient are shown in Figure 4. In the left column are the results from one of the control eyes (subject AG, right eye). The trace arrays and response topographies of Figures $4 \mathrm{~B}$ and $\mathrm{C}$ represent the distributions of the direct response (epoch $0-50 \mathrm{~ms}$ ) and the induced component (epoch 50-80 ms) respectively. A scalar product response density estimate ${ }^{9}$ computed with templates obtained from the rings showed in the inset in Figure 4. With the 1:2 ratio of multifocal and global flash intensities used in this study, the direct response tends to be larger than the induced component. The drop off with eccentricity of both components is qualitatively similar to that produced by a standard multifocal flicker stimulus ${ }^{9}$; however, the induced component exhibits a pronounced nasal temporal asymmetry at about $5^{\circ}$ eccentricity. Note the depression in the temporal hemifield at the blind spot.

The right column of Figure 4 shows results obtained from the right eye of LA, a 48 year old diabetic woman with a 12 year history of IDDM. Although ophthalmoscope and slit lamp examinations showed no abnormalities, amplitudes of the direct responses are markedly reduced compared with those of the control subject (left column). In this diabetic, amplitudes of the induced component were larger than those of the direct responses. However, owing to large intersubject variability of the induced component, it appears to be less reliable as an indicator of disease than the direct response. A dip in the induced component topography is seen in the temporal field at around $5^{\circ}$ eccentricity. This topographic feature was also observed in a number of controls and is, therefore, not considered to be a characteristic of retinal dysfunction.

\section{STATISTICAL ANALYSIS}

Before comparing diabetics with controls, we checked the effect of age on the response amplitudes of the control subjects. To this end the responses from all 103 elements were summed in each record. We also analysed responses individually and in regional averages derived from rings, quadrants, and hemifields with similar results. The amplitudes of the resulting wave forms are plotted for all control subjects in Figure 5A. The direct response amplitudes were negatively correlated with age $(r=-0.663, \mathrm{p}<0.001)$. Diabetic subjects also showed a reduction of direct response amplitude due to age (right eye: $r=-0.739, \mathrm{p}<0.01$, left eye: $r=-0.712, \mathrm{p}=0.014$; plots are not shown). Thus, response amplitudes should only be evaluated as an indicator of functional changes in diabetics if they are compared with age matched controls. Our sample size was too small to permit data analysis in multiple age groups. However, as a first approximation to age matching, we restricted the comparison to subjects between 35 and 70 years of age (indicated by the band between the broken lines in Fig 5A). In this age group, nine control subjects (50.9 (SD 10.3) years old) and 10 diabetics (52.3 (9.2) years old) were included.

In the 35-70 year old group, the direct response was significantly smaller in diabetics than in control subjects, as shown in Figure 6A (control eyes: $\mathrm{n}=9,21.0$ (5.4) $\mu \mathrm{V}$; diabetic right eyes: $\mathrm{n}=10,15.3(4.8) \mu \mathrm{V} ; \mathrm{p}=0.028$; diabetic left eyes: $\mathrm{n}=10,13.6(4.7) \mu \mathrm{V} ; \mathrm{p}$ $<0.01)$. The range of direct response amplitudes in the control eyes was 14.5-29.7 $\mu \mathrm{V}$. The number of diabetic eyes whose amplitudes fell outside (below) this range was five of nine right eyes and six of nine left eyes.

There was a marginal tendency for the amplitudes of the patients' direct responses to be associated with longer histories of diabetes. However, this tendency was not significant in either single eye analysis (right eye: $\mathrm{n}=10, r$ $=0.483, \mathrm{p}=0.133$, left eye: $\mathrm{n}=10, r=0.402, \mathrm{p}$ $=0.221$; plots are not shown). Since age and 


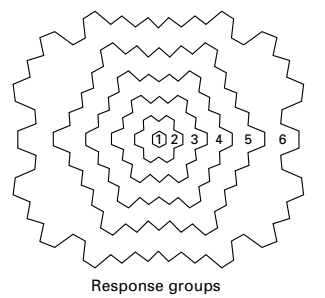

A

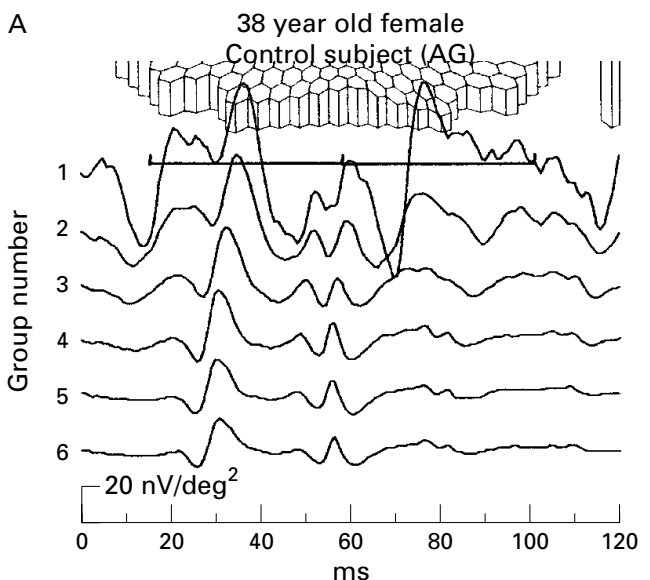

B

Direct responses (0-50 ms)

ar man are now un an

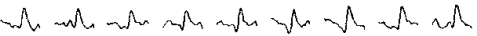

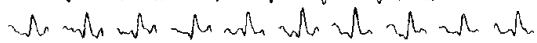

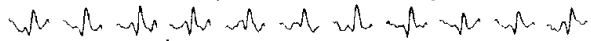

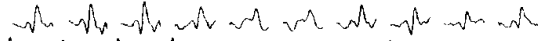

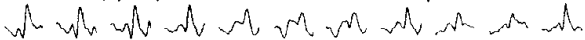

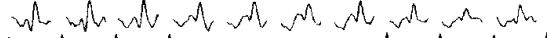

wh wh wh wh wh and at wh wh

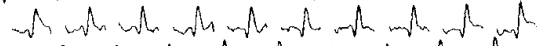

$0.5 \mu \mathrm{V}{ }_{0} 50 \mathrm{~ms}$ of $\mathrm{N}$.

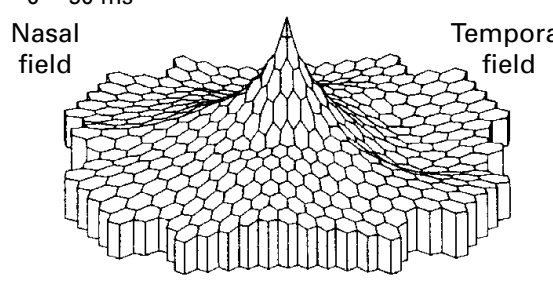

C

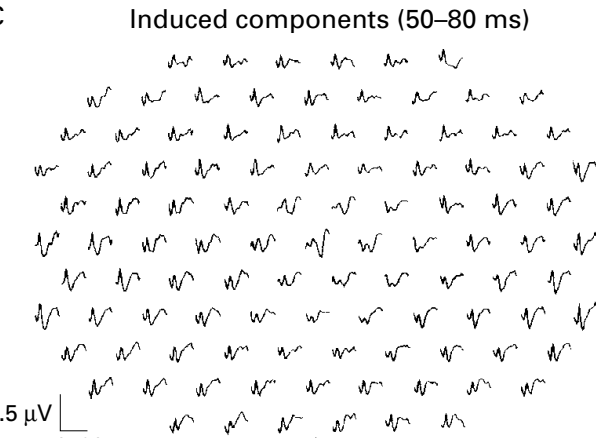

$80 \mathrm{~ms}$
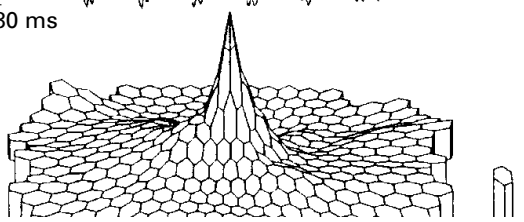

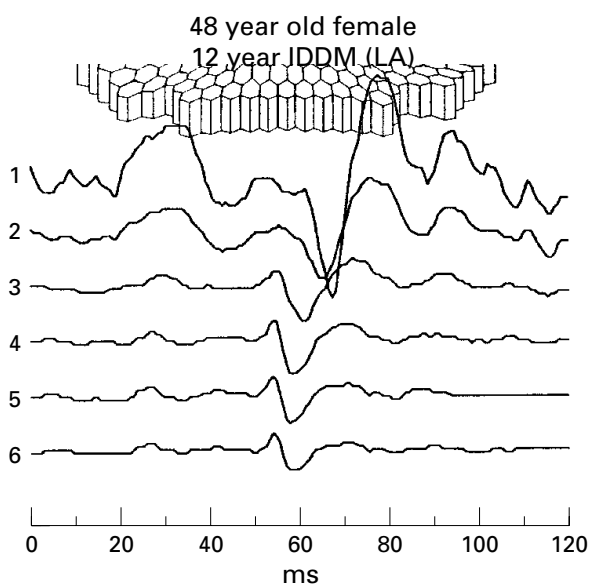

Direct responses (0-50 ms)

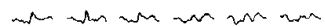

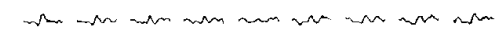

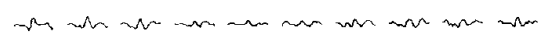

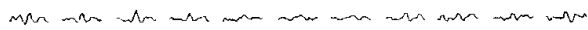

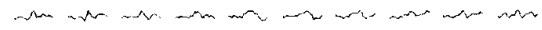

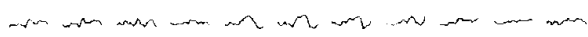

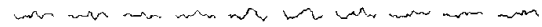

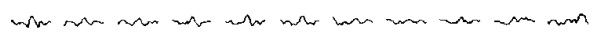

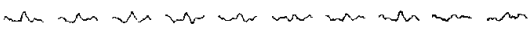

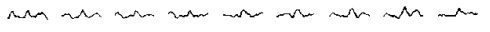

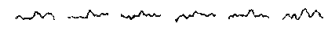

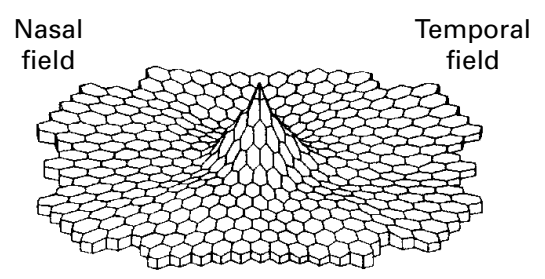

Induced components (50-80 ms)

vin $n$ w $n$

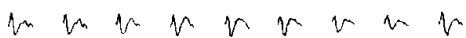

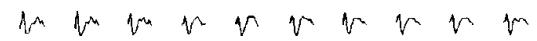

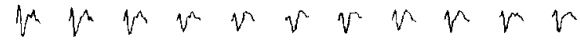

$\mu r r r r r$

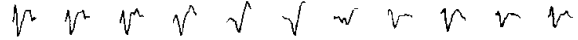
$r \mu r v r r a r r$ $m p r v r r q r q m$

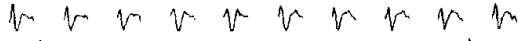

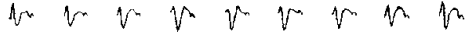

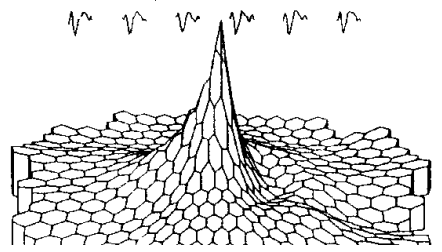

$20 \mathrm{nV} / \mathrm{deg}^{2}$

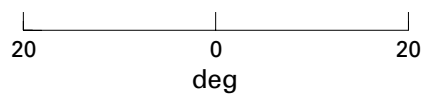

Figure 4 Data recorded from a 38 year old female control subject (left column), and a 48 year old female patient with a 12 year history of IDDM (right column). (A) Traces averaged over the concentric rings shown in inset ( $B$ and $C)$. Trace arrays and topographies of direct response (B) and induced component $(C)$. 
A
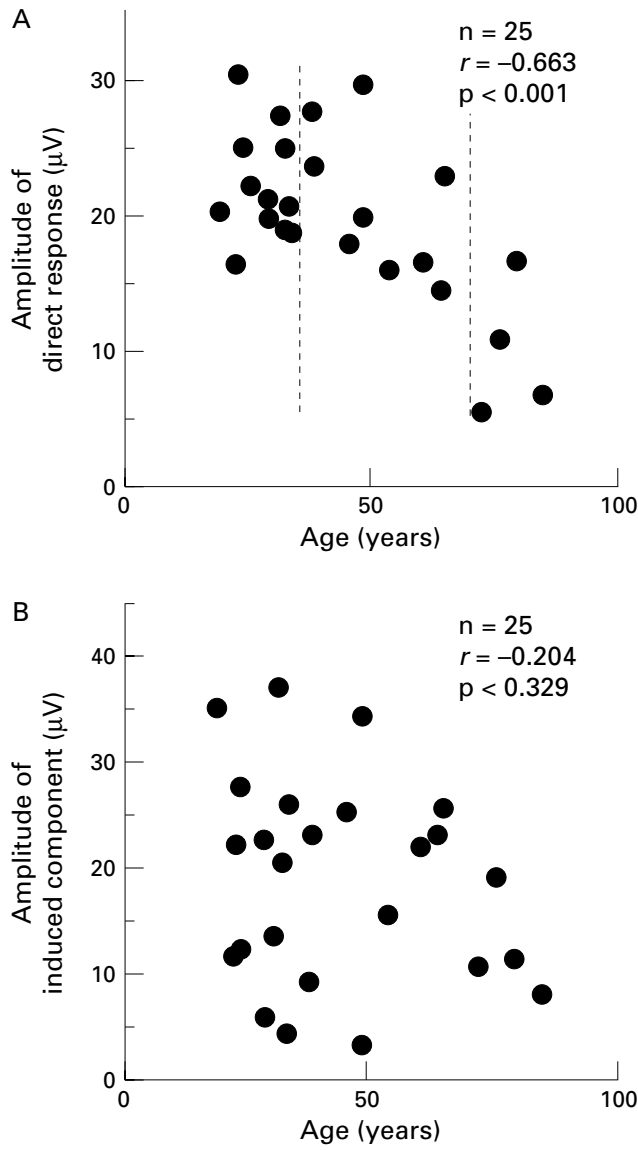

Figure 5 Relation between response amplitudes and age in control subjects. (A) Direct response. (B) Induced component. The responses from all 103 hexagons were added and then the amplitudes were measured. The data between the broken lines in $(A)$ (ages 35-70 years) were used when comparing diabetics and control subjects.

duration of diabetes are not independent variables in this study, no firm conclusion concerning either variable could be drawn. We also analysed IDDM and NIDDM independently in the effect of age and duration of disease. However, the small sample size of each subgroup did not permit any conclusion.

The induced component amplitudes did not correlate with age in the control group ( $\mathrm{p}$ $>0.3$ ). In addition, there was a 10 -fold range in the amplitude of this component within this group. Therefore, any effect diabetes might have on this component was overshadowed by this intersubjective variability. In the age group 35-70, the mean amplitudes (SD) were 20.5 (9.4) $\mu \mathrm{V}$ (controls), 20.7 (12.3) $\mu \mathrm{V}$ (diabetic group, right eye; $\mathrm{p}>0.9$ ), and $17.3(10.8) \mu \mathrm{V}$ (diabetic group, left eye; $p>0.5$ ). These results are shown in Figure 6B. A more detailed analysis of response averages over concentric rings also revealed no significant differences between controls and diabetics.

We also analysed the implicit times of the two response components. No significant differences were found between the groups in the implicit times of the direct response or the induced component.

\section{Discussion}

We focused on the detection of functional changes in the retina that precede diabetic
A

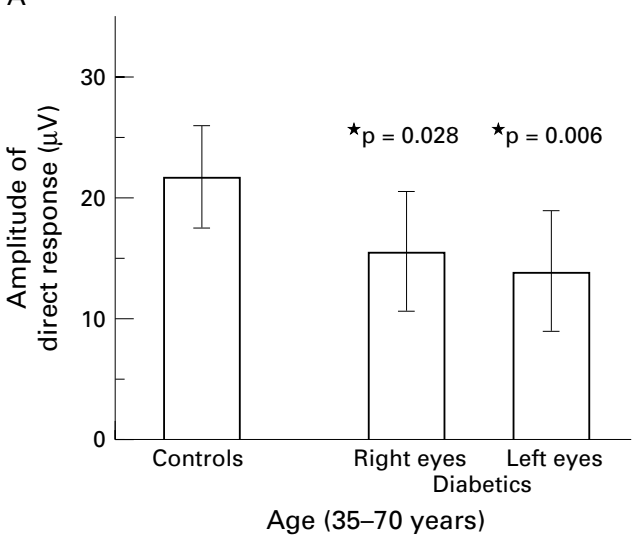

B

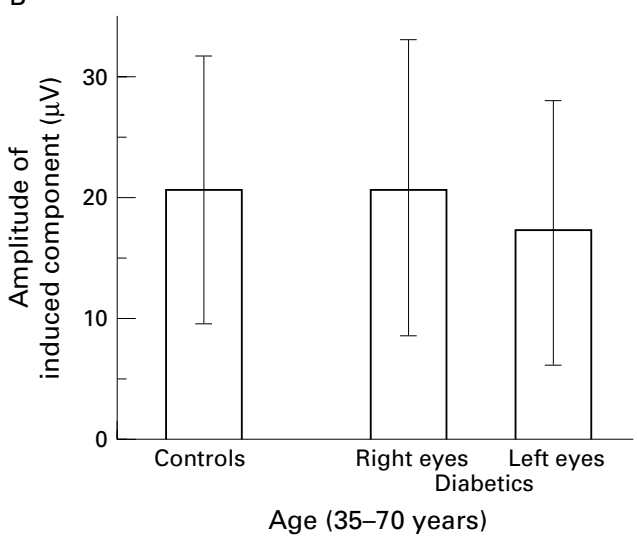

Figure 6 Comparison of response amplitudes between diabetics and controls. In both panels, age was limited to 35-70 years. (A) The direct response was significantly different (control eyes: $n=9,21.0$ (5.4) $\mu \mathrm{V}$; diabetics right eyes: $n=10,15.3$ (4.8) $\mu \mathrm{V} ; p=0.028$; diabetics left eyes: $n=10,13.6$ (4.7) $\mu V ; p<0.01)$. (B) There was no significant difference in the induced component between the two groups (control eyes: $n=9,20.5$ (9.4) $\mu \mathrm{V}$; diabetics right eyes: $n=10,20.7$ (12.3) $\mu \mathrm{V} ; p>0.9$; diabetics left eyes: $n=10,17.3(10.8) \mu V ; p>0.5)$.

retinopathy, as the patients included in this study showed no pathological changes detectable by standard ophthalmoscopy. Since sensitive invasive tests such as fluorescein angiography were not clinically indicated and were therefore not used, it is not possible to guarantee a complete absence of retinopathy. The direct response revealed a significant amplitude reduction in diabetes. It is also sensitive to the age of healthy control subjects, so age matched control and patient groups were utilised.

Among the subjects in the diabetic group, one might expect a large range of latent diabetic changes. Duration of the disease is one of the most important risk factors for retinopathy. It was, therefore, somewhat unexpected to find that the amplitude reduction in the direct response was not significantly correlated with the duration of diabetes. This may be explained in part by the fact that onset of disease and the date of diagnosis may differ widely. However, there were not sufficient numbers of patients of different ages to draw a definite conclusion.

There was a large range in the amplitude of the induced component among both control 
and diabetic subjects, and a significant difference between groups was not found. A recent parametric study found that a dim focal flash $\left(0.33-0.67 \mathrm{~cd} / \mathrm{s} / \mathrm{m}^{2}\right)$ appears to produce induced components with less intersubjective variability. However, under these stimulus conditions the signal to noise ratio of the direct response is low.

It is interesting that the two response components investigated in this study were not equally affected by diabetes. To understand this finding better we need to consider the difference in the generation of them. The responses to the multifocal stimuli and those to the interleaved global flashes can mutually affect each other. However, these interactions manifest themselves differently in the direct response and induced component. The direct response is produced by the focal flash, which is always preceded $26.7 \mathrm{~ms}$ earlier by a brighter global flash. We would expect this component to be reduced if the recovery from the preceding flash is impaired or light adaptation produced a large desensitisation. The induced component, on the other hand, is the change in the global flash response produced by the preceding focal flash. It is a second order component that does not reflect the size of the global flash response directly, but rather the response's recovery from the preceding focal flash. Our results suggest that diabetics have a larger than normal reduction in the amplitude of the focal responses produced by the bright periodic (global) flashes. However, the disease does not significantly affect recovery of the global flash response from the weaker focal flashes during the $26.7 \mathrm{~ms}$ interflash interval under the conditions examined here.

The conclusion that early effects of diabetes are predominantly reflected in interactions between consecutive flash responses was also reported by Palmowski et $a l^{10}$ in a previous multifocal ERG study. In a double flash ERG study, Gliem et al found a larger reduction in the responses to the second flash in diabetics compared with control subjects. ${ }^{3}$ While a single, global stimulus and a longer mean interflash interval $(120 \mathrm{~ms})$, were used in their study, their findings are also consistent with the present results.

The authors thank Alex Lomakin for software development. This work was supported by the Smith-Kettlewell Eye Research Institute, the Juvenile Diabetes Foundation grant 5-1998-6, and Institute, the Juvenile

1 Yonemura D, Aoki T, Tsuzuki K. Electroretinogram in diabetic retinopathy. Arch Ophthalmol 1962;68:19-24.

2 Simonsen SE. Prognostic value of ERG (oscillatory potential) in juvenile diabetics. Acta Ophthalmol 1973; 23(suppl): 223-4.

3 Gliem H, Möller DE, Kietzmann G. Das Doppelblitz-ERG bei der diabetischen Retinopathie. Acta Ophthalmol 1973; 51:85-94.

4 Coupland SG. Pattern ERG and oscillatory potential measures in diabetic retinopathy. [ARVO Abstracts]. Invest Ophthalmol Vis Sci 1985;26:S215 (Abstract no 19).

5 Juen S, Kieselbach GF. Electrophysiological changes in juvenile diabetics without retinopathy. Arch Ophthalmol 1990;108:372-5.

6 Yoshida A, Kojima M, Ogasawara $\mathrm{H}$, et al. Oscillatory potentials and permeability of the blood-retinal barrier in noninsulin-dependent diabetic patients without retinopathy. Ophthalmology 1991;98:1266-71.

7 Wanger P, Persson HE. Early diagnosis of retinal changes in diabetes: a comparison between electroretinography and diabetes: a comparison between electroretinography and
retinal biomicroscopy. Acta Ophthalmol 1985;63:716-20.

retinal biomicroscopy. Acta Ophthalmol 1985;63:716-20.
Arden GB, Hamilton AM, Wilson-Holt J, et al. Pattern electroretinograms become abnormal when background diabetic retinopathy deteriorates to a preproliferative stage: possible use as a screening test. $\mathrm{Br} \quad \mathcal{F}$ Ophthalmol 1986;70:330-5.

9 Sutter EE, Tran D. The field topography of ERG components in man- $\mathrm{I}$. The photopic luminance response. Vis Res 1992;32:433-46.

10 Palmowski AM, Sutter EE, Bearse MA, et al. Mapping of retinal function in diabetic retinopathy using the multifocal electroretinogram. Invest Ophthalmol Vis Sci 1997;38:258696.

11 Fortune B, Schneck ME, Adams AJ. Delayed multifocal electroretinograms reveal local retinal dysfunction in diabetic persons with and without retinopathy. Invest Ophdiabetic persons with and without
thalmol Vis Sci 1999;40:2638-51.

12 Sutter EE, Bearse MA. The retinal topography of local and lateral gain control mechanisms. Vision science and its applications. Technical Digest Series. Vol 1. Washington, DC: Optical Society of America, 1998;20-3.

13 Sutter EE, Shimada Y, Li Y, et al. Mapping inner retinal function through enhancement of adaptive components in the M-ERG. Vision Science and its applications. Technical Digest Series. Washington, DC: Optical Society of America, $1999 ; 52-5$ 